Louisiana State University

LSU Digital Commons

Faculty Publications

Department of Physics \& Astronomy

$12-1-2004$

\title{
Seismic isolation and suspension systems for advanced LIGO
}

\author{
N. A. Robertson \\ Ginzton Laboratory \\ B. Abbott \\ California Institute of Technology \\ R. Abbott \\ LIGO Livingston \\ R. Adhikari \\ LIGO, Massachusetts Institute of Technology \\ G. Allen \\ Ginzton Laboratory
}

See next page for additional authors

Follow this and additional works at: https://digitalcommons.Isu.edu/physics_astronomy_pubs

\section{Recommended Citation}

Robertson, N., Abbott, B., Abbott, R., Adhikari, R., Allen, G., Armandula, H., Aston, S., Baglino, A., Barton, M., Bland, B., Bork, R., Bogenstahl, J., Cagnoli, G., Campbell, C., Cantley, C., Carter, K., Cook, D., Coyne, D., Crooks, D., Daw, E., DeBra, D., Elliffe, E., Faludi, J., Fritschel, P., Ganguli, A., Giaime, J., Gossler, S., Grant, A., Greenhalgh, J., Hammond, M., Hanson, J., Hardham, C., \& Harry, G. (2004). Seismic isolation and suspension systems for advanced LIGO. Proceedings of SPIE - The International Society for Optical Engineering, 5500, 81-91. https://doi.org/10.1117/12.552469

This Conference Proceeding is brought to you for free and open access by the Department of Physics \& Astronomy at LSU Digital Commons. It has been accepted for inclusion in Faculty Publications by an authorized administrator of LSU Digital Commons. For more information, please contact ir@lsu.edu. 


\section{Authors}

N. A. Robertson, B. Abbott, R. Abbott, R. Adhikari, G. Allen, H. Armandula, S. Aston, A. Baglino, M. Barton, B. Bland, R. Bork, J. Bogenstahl, G. Cagnoli, C. Campbell, C. A. Cantley, K. Carter, D. Cook, D. Coyne, D. Crooks, E. Daw, D. DeBra, E. Elliffe, J. Faludi, P. Fritschel, A. Ganguli, J. Giaime, S. Gossler, A. Grant, J. Greenhalgh, M. Hammond, J. Hanson, C. Hardham, and G. Harry 


\title{
PROCEEDINGS OF SPIE
}

\section{Seismic isolation and suspension systems for Advanced LIGO}

\author{
Norna A. Robertson, Benjamin Abbott, R. Abbott, R. \\ Adhikari, Graham S. Allen, et al.
}

Norna A. Robertson, Benjamin Abbott, R. Abbott, R. Adhikari, Graham S. Allen, Helena Armandula, Stuart Mark Aston, A. Baglino, Mark Barton, B. Bland, Rolf Bork, J.

Bogenstahl, Gianpietro Cagnoli, C. Campbell, C. A. Cantley, K. Carter, D. Cook, D. Coyne, David R.M. Crooks, Edward J. Daw, Daniel B. DeBra, E. Elliffe, J. Faludi, Peter Fritschel, A. Ganguli, Joseph A. Giaime, S. Gossler, A. Grant, J. Greenhalgh, M. Hammond, Jonathan Hanson, C. Hardham, Gregory M. Harry, Alistair Heptonstall, Jay Heefner, James Hough, D. Hoyland, Wensheng Hua, L. Jones, R. Jones, Jonathan E. Kern, J. LaCour, Brian T. Lantz, K. Lilienkamp, N. Lockerbie, Harald Lueck, M. Maclnnis, K. Mailand, Ken Mason, R. Mittleman, Samir A. Nayfeh, J. Nichol, David J. Ottaway, H. Overmier, M. Perreur-Lloyd, J. Phinney, M. V. Plissi, W. Rankin, D. I. Robertson, J. Romie, Sheila Rowan, R. Scheffler, David H. Shoemaker, P. Sarin, Peter H. Sneddon, Clive C. Speake, O. Spjeld, G. Stapfer, Kenneth A. Strain, C. I. Torrie, G. Traylor, J. van Niekerk, Alberto Vecchio, Shirong Wen, P. Willems, I. Wilmut, Harry Ward, M. Zucker, Lei Zuo, "Seismic isolation and suspension systems for Advanced LIGO," Proc. SPIE 5500, Gravitational Wave and Particle Astrophysics Detectors, (29 September 2004); doi: 10.1117/12.552469

Event: SPIE Astronomical Telescopes + Instrumentation, 2004, Glasgow, United Kingdom 


\title{
Seismic isolation and suspension systems for Advanced LIGO
}

N. A. Robertson*,a,b, B Abbott ${ }^{\mathrm{c}}$, R. Abbott ${ }^{\mathrm{d}}$, R. Adhikari ${ }^{\mathrm{e}}$, G. Allen ${ }^{\mathrm{a}}$, H. Armandula ${ }^{\mathrm{c}}$, S. Aston ${ }^{\mathrm{f}}$, A. Baglino ${ }^{\mathrm{a}}$, M. Barton ${ }^{\mathrm{c}}$, B. Bland ${ }^{\mathrm{g}}$, R. Bork ${ }^{\mathrm{c}}$, J. Bogenstahl $^{\mathrm{h}, \mathrm{b}}$, G. Cagnoli ${ }^{\mathrm{b}}$, C. Campbell ${ }^{\mathrm{a}}$,

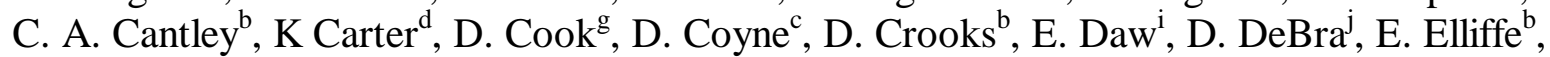
J. Faludi ${ }^{\mathrm{a}}$, P. Fritschel ${ }^{\mathrm{e}}$, A. Ganguli ${ }^{\mathrm{a}}$, J. Giaime $^{\mathrm{i}, \mathrm{d}}$, S. Gossler ${ }^{\mathrm{h}}$, A. Grant ${ }^{\mathrm{b}}$, J. Greenhalgh $^{\mathrm{k}}$,

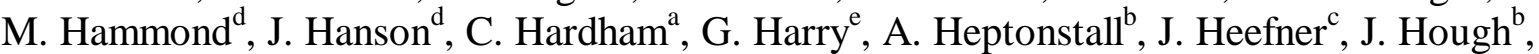
D. Hoyland ${ }^{\mathrm{f}}$, W. Hua ${ }^{\mathrm{a}}$, L. Jones ${ }^{\mathrm{c}}$, R. Jones ${ }^{\mathrm{b}}$, J. Kern ${ }^{\mathrm{d}}$, J. LaCour ${ }^{\mathrm{d}}$, B. Lantz ${ }^{\mathrm{a}}$, K. Lilienkamp ${ }^{\mathrm{e}}$,

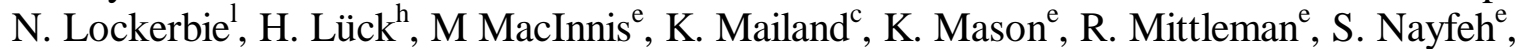
J. Nichol ${ }^{\mathrm{a}}$, D. J. Ottaway ${ }^{\mathrm{e}}$, H. Overmier ${ }^{\mathrm{d}}$, M. Perreur-Lloyd ${ }^{\mathrm{b}}$, J. Phinney ${ }^{\mathrm{e}}$, M. Plissi ${ }^{\mathrm{b}}$, W. Rankin ${ }^{\mathrm{e}}$, D. Robertson $^{\mathrm{b}}$ J. Romie ${ }^{\mathrm{c}}$, S. Rowan ${ }^{\mathrm{b}}$, R. Scheffler ${ }^{\mathrm{a}}$, D. H. Shoemaker ${ }^{\mathrm{e}}$, P Sarin ${ }^{\mathrm{e}}$, P. Sneddon ${ }^{\mathrm{b}}$, C. Speake, O. Spjeld ${ }^{\mathrm{f}}$, G. Stapfer ${ }^{\mathrm{d}}$, K. A. Strain ${ }^{\mathrm{b}}$, C. Torrie ${ }^{\mathrm{c}}$, G. Traylor ${ }^{\mathrm{d}}$, J. van Niekerk ${ }^{\mathrm{a}}$,

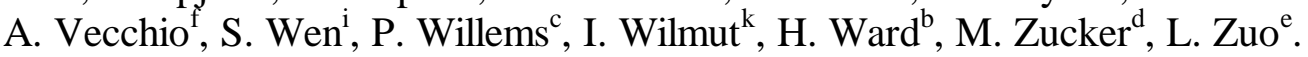

${ }^{a}$ Edward L Ginzton Laboratory, Stanford University, Stanford CA 94305, USA;

bInstitute for Gravitational Research, University of Glasgow, Glasgow G12 8QQ, Scotland, UK;

${ }^{c}$ LIGO Laboratory, California Institute of Technology, MS 18-34, Pasadena, CA 91125, USA;

${ }^{\mathrm{d}}$ LIGO Livingston Observatory, Box 940, Livingston, LA 70754, USA;

eLIGO Laboratory, Bldg. NW17-161, Massachusetts Institute of Technology, Cambridge, MA 02139, USA;

${ }^{\mathrm{f}}$ School of Physics and Astronomy, University of Birmingham, Edgbaston, Birmingham, B15 2TT, UK;

${ }^{\mathrm{g}}$ LIGO Hanford Observatory, Box 159, Richland, WA, 99352 USA;

${ }^{\mathrm{h}}$ Universitat Hannover, Institut für Atom und Molekülphysik, Abteilung Spektroskopie, D30167, Hannover, Germany;

${ }^{\mathrm{i}}$ Department of Physics and Astronomy, Louisiana State University, Baton Rouge, LA 70803, USA;

${ }^{\mathrm{j}}$ Department of Aeronautics and Astronautics, Stanford University, Stanford CA 95305, USA;

${ }^{k}$ Engineering Department, Rutherford Appleton Laboratory, Chilton, Didcot, Oxon OX11 0QX;

${ }^{1}$ Department of Physics and Applied Physics, University of Strathclyde, Glasgow G4 0NG, Scotland, UK.

\begin{abstract}
To meet the overall isolation and alignment requirements for the optics in Advanced LIGO, the planned upgrade to LIGO, the US laser interferometric gravitational wave observatory, we are developing three sub-systems: a hydraulic external pre-isolator for low frequency alignment and control, a two-stage active isolation platform designed to give a factor of $\sim 1000$ attenuation at $10 \mathrm{~Hz}$, and a multiple pendulum suspension system that provides passive isolation above a few hertz. The hydraulic stage uses laminar-flow quiet hydraulic actuators with millimeter range, and provides isolation and alignment for the optics payload below $10 \mathrm{~Hz}$, including correction for measured Earth tides and the microseism. This stage supports the in-vacuum two-stage active isolation platform, which reduces vibration using force feedback from inertial sensor signals in six degrees of freedom. The platform provides a quiet, controlled structure to mount the suspension system. This latter system has been developed from the triple pendulum suspension used in GEO 600 , the German/UK gravitational wave detector. To meet the more stringent noise levels required in Advanced LIGO, the baseline design for the most sensitive optics calls for a quadruple pendulum, whose final stage consists of a $40 \mathrm{~kg}$ sapphire mirror suspended on fused silica ribbons to reduce suspension thermal noise.
\end{abstract}

*nornar@stanford.edu, phone 650723 0228, fax 6507232666 
Keywords: gravitational wave detection, seismic isolation, suspension system, quiet hydraulic system, active seismic isolation platform, silica ribbon suspension

\section{INTRODUCTION}

The initial LIGO detectors ${ }^{1,2}$ which are currently being commissioned use the principle of the Michelson interferometer to sense differential changes in two perpendicular arm lengths to search for strains in space produced by gravitational waves of astrophysical origin. By the time it has completed its series of science data runs, Initial LIGO may have detected gravitational waves. However an order of magnitude increase in broadband sensitivity and an extension of the operational bandwidth to lower frequencies would greatly increase the probability of detection and offer exciting opportunities for carrying out gravitational wave astronomy. An upgrade to LIGO, Advanced LIGO ${ }^{3,4}$, has been proposed to achieve such an improvement in performance, and members of the LIGO Scientific Collaboration are working with the LIGO Laboratory in carrying out research towards developing a design which would result in such an increase in sensitivity. The sensitivity of the current LIGO detectors is expected to be limited by thermal noise associated with the suspensions of its mirrors at frequencies in the region $\sim 40 \mathrm{~Hz}$ to $\sim 150 \mathrm{~Hz}$. Below $40 \mathrm{~Hz}$ the dominant noise source is expected to be residual seismic noise, filtered by the combination of a 4 layer passive isolation stack and a single pendulum suspension. To improve the sensitivity in Advanced LIGO such that its useful working range extends down to $\sim 10 \mathrm{~Hz}$ requires both a reduction of suspension thermal noise and an improvement in seismic isolation. In this paper we discuss how we propose to achieve such improvements, and summarize the work to date.

To achieve the overall suspension, isolation and alignment requirements for Advanced LIGO, we are developing three sub-systems:

- a hydraulic external pre-isolator system (HEPI) for low frequency alignment and control, which will be situated outside the vacuum system

- a two-stage in-vacuum active isolation platform designed to give a factor of $\sim 1000$ attenuation at $10 \mathrm{~Hz}$

- a multiple pendulum suspension system (quadruple for the most sensitive optics) that provides passive isolation above a few hertz, and minimizes suspension thermal noise by using silica ribbons in the final stage.

In Fig. 1 we show a schematic diagram of the layout of these three sub-systems in the largest of the vacuum chambers in which will be suspended the most sensitive optics in the interferometers. We discuss each of these sub-systems in the following sections.

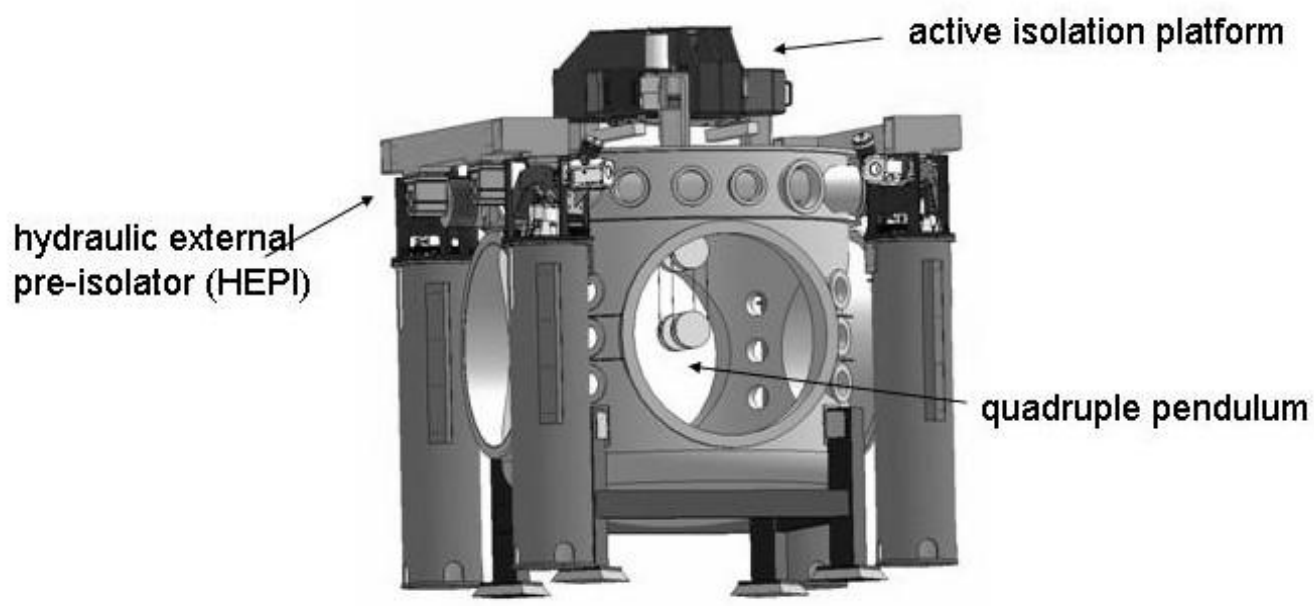

Figure 1. Schematic diagram of the overall suspension and isolation system proposed for use in Advanced LIGO. The three main subsystems are indicated. The top of the vacuum system and the doors are removed for clarity. A supporting structure for the quadruple pendulum, which would be rigidly attached to the isolation platform, is also not shown. For scale, the height of the supporting piers to the base of HEPI is $\sim 2.5 \mathrm{~m}$. 


\section{HYDRAULIC EXTERNAL PRE-ISOLATOR}

The hydraulic external pre-isolator (HEPI) system was specifically designed to address the low frequency isolation and alignment requirements for Advanced LIGO. Actuation is required in all six degrees of freedom (DOF), and the specifications for this system are that it should be able to generate a maximum force greater than $2000 \mathrm{~N}$ and a throw of $+/-1 \mathrm{~mm}$, have a bandwidth from 0 to $\sim 10 \mathrm{~Hz}$, and a noise level not exceeding $10^{-9} \mathrm{~m} / \sqrt{ } \mathrm{Hz}$ at $1 \mathrm{~Hz}$. A quiet hydraulic actuator can meet all of these requirements. In such a system the flow is not permitted to become turbulent, and thus by maintaining laminar flow throughout the system, a quiet hydraulic actuator can reach very low noise levels.

A schematic diagram of the basic elements of the system is shown in Fig. 2. Its basic mode of operation is described in the figure caption. Further details of the design and operation of the hydraulic system are given elsewhere. ${ }^{5}$

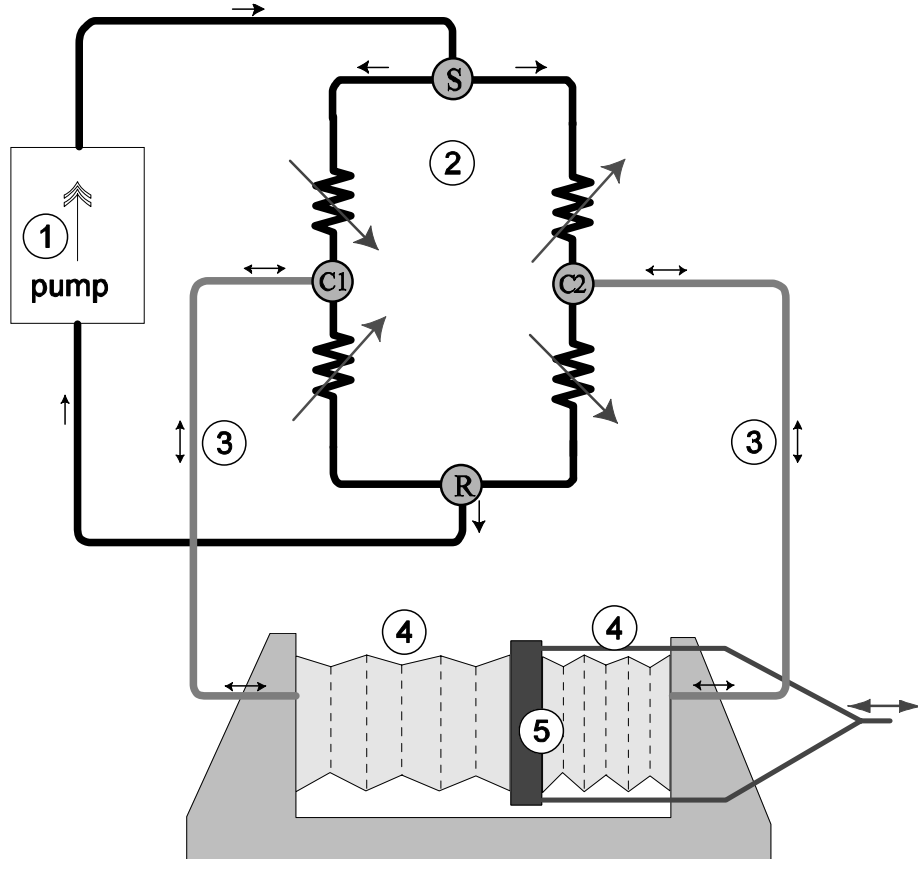

Figure 2. A schematic diagram of a quiet hydraulic actuator is shown on the left. The pump, (1), supplies a constant flow of fluid through the actuator. This fluid flows continuously through the hydraulic equivalent of a Wheatstone bridge (2), with variable resistances that are controlled in differential pairs. By controlling the resistance, one generates differential pressure across the bridge, which modifies the flow, (3), to the differential bellows, (4). These bellows act as a stiction-free piston which moves the actuator plate, (5), which is connected to the payload (not shown) with a flexure stiff in 1 DOF.

The performance requirements for LIGO include alignment and isolation. To achieve both of these requirements, two controls techniques are used, namely sensor blending and sensor correction. In this application each actuator is outfitted with a displacement sensor which measures the difference between the actuator plate position and the ground, and a passive $1 \mathrm{~Hz}$ seismometer. These two signals are blended together into a "supersensor" in feedback, it is possible to control position at low frequency while still attaining isolation at higher frequencies. The isolation can be extended to lower frequencies using sensor correction, which can be described as the subtraction of an undesired signal from a sensor output. At frequencies where the displacement sensor signal dominates, the feedback loop achieves no isolation. By suitably adding the signal from a ground-mounted low-frequency seismometer to the displacement sensor output, the resulting signal can then be used to null the actuator plate position and hence provide isolation.

In the LIGO application there will be 8 actuators used to control the 6 DOFs, 4 horizontal and 4 vertical mounted in pairs on each of the 4 piers supporting the payload in the vacuum tanks (as indicated in Fig. 1). Actuation can be used to track the Earth's tides, as well as to correct at each vacuum tank for large amplitude low-frequency $(\sim 0.1 \mathrm{~Hz}$ to several hertz) motion including the microseism which typically peaks at frequencies near $0.15 \mathrm{~Hz}$. 
Following extensive development and testing of the actuator design at Stanford University, prototype actuators were installed and tested on a LIGO-sized vacuum chamber at the LIGO Advanced System Test Interferometer (LASTI) facility at MIT. This system showed good performance in the three displacement DOFs, reducing motion between several tenths of hertz to a few hertz, achieving about an order of magnitude noise reduction between $0.5 \mathrm{and} 2 \mathrm{~Hz}$. An example of the performance obtained is shown in Fig. 3.

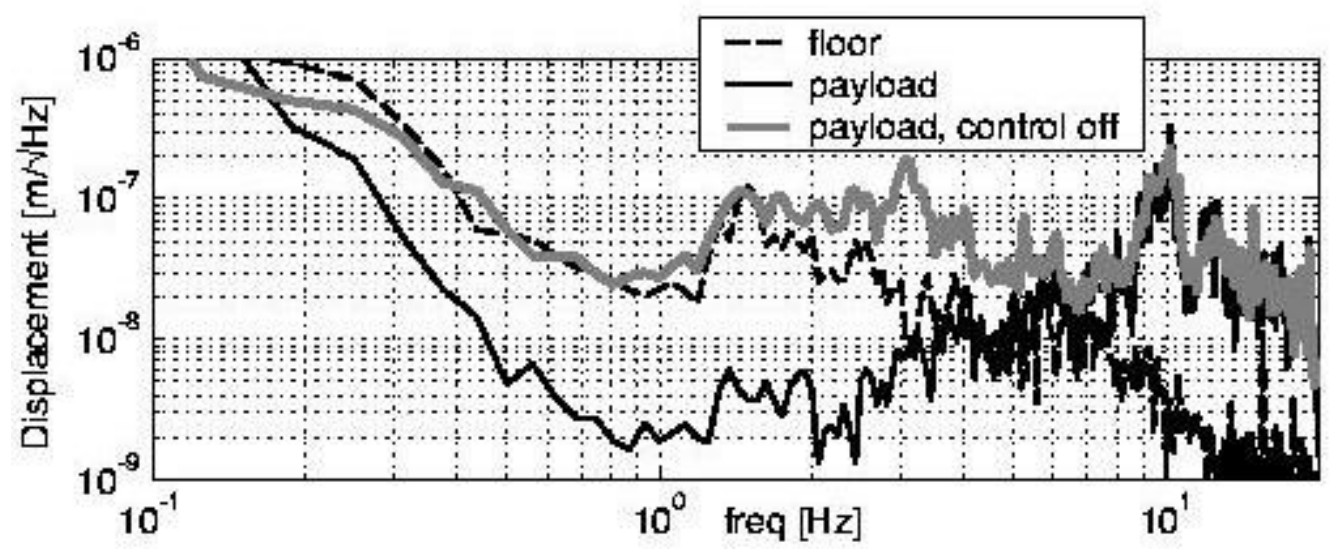

Figure 3. Performance of the prototype HEPI system. The black and gray lines are the horizontal (beam direction) motion at the payload, with and without the noise-reduction system. A factor of approximately 10 noise reduction is seen over the band $0.5-2 \mathrm{~Hz}$. The dashed line is the motion of the floor near the system.

Although the original intention was for installation of HEPI in LIGO in a few years time when the system is upgraded, over the past year the development has been accelerated to allow for installation at the LIGO Livingston site this year (2004). This decision was taken in order to allow reliable detector operation during high-noise periods, such as the daylight hours of weekdays, when excess ground motion due to human activity has been found to be a serious problem. Installation of HEPI is underway at the time of writing (Spring 2004). A picture showing the installation in progress at one of the large vacuum chambers at LIGO Livingston is shown in Fig. 4.

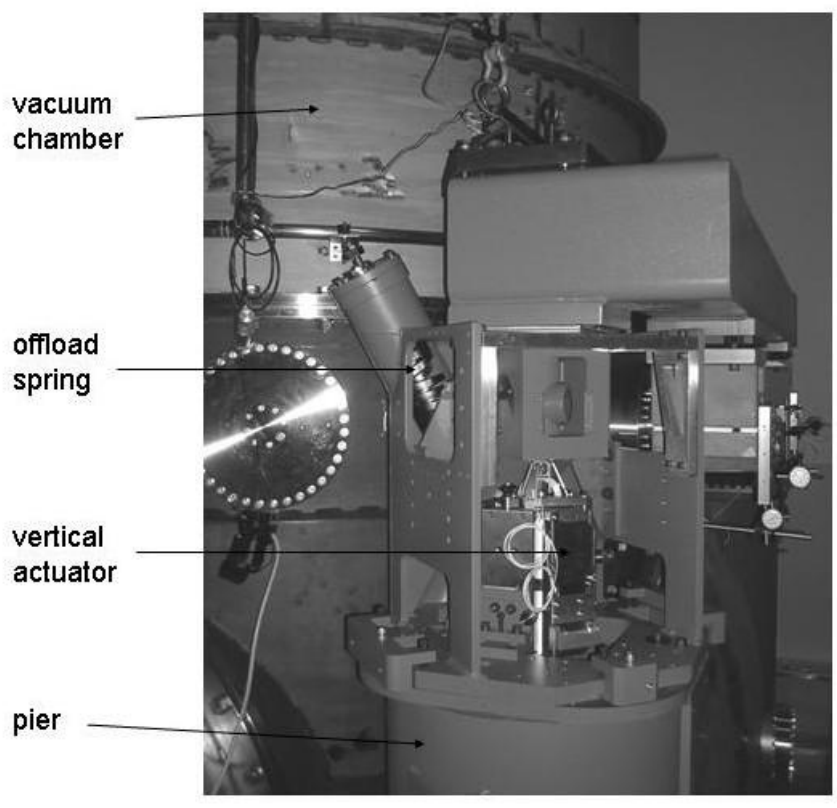

Figure 4. HEPI unit being installed on one pier of a vacuum chamber at the LIGO Livingston site. One of the two offload springs which support the payload at this corner is indicated. 


\section{ACTIVE PLATFORM DEVELOPMENT}

We have discussed above the first stage of isolation for Advanced LIGO, which is situated outside the vacuum system. This outer stage will support the in-vacuum two-stage active isolation platform which we now describe. This stage can be thought of as a direct replacement for the 4 layer passive isolation stack currently in use in LIGO. The basic design strategy for the isolation platform has been discussed in earlier papers ${ }^{7,8}$. Here we present a brief overview of the design features, review the current status of development and present recent data from the prototype platform being studied at Stanford University. One of the most challenging problems for achieving good seismic isolation at low frequencies is that of tilt-horizontal coupling, which is introduced because horizontal inertial sensors cannot distinguish between horizontal acceleration and tilt. How this problem is tackled in the control of the isolation system is addressed in another paper $^{9}$ in this volume, and thus will not be covered in detail here.

The isolation platform consists of two cascaded stages, suspended through stiff blade springs and short pendulum links, giving natural frequencies in the $2-10 \mathrm{~Hz}$ range. The vibration of each stage is reduced by sensing its motion in 6 degrees of freedom (DOFs) and applying forces in feedback loops to reduce the sensed motion. The feedback signal for the first stage is derived by blending signals from three sensors for each DOF - a long-period broadband seismometer (Streckeisen STS-2), a short-period geophone and a relative position sensor. The second stage uses signals from a GS-13 (Geotech Instruments) low-noise geophone and a relative position sensor for each DOF. The actuators are electromagnetic non-contacting forcers, which apply forces between the support and stage one, and between stage one and stage two respectively. An advantage of a fairly stiff suspension is its low thermal sensitivity. With feedback applied, the system is further stiffened, and this provides a high impedance mechanical interface between the platform and any element suspended from it.

Two mechanical designs are required for the two different vacuum chamber designs in LIGO. In the chambers housing the most sensitive optics the active platform is situated toward the top of the chamber (see Fig 1) and the suspension attaches from below to an interface surface (optics table). In the smaller chambers the active platform is located lower down in the chamber, and the suspension system will sit in its support structure on top of the optics table. A technology demonstrator isolation platform is currently being investigated at Stanford. It is shown in Fig 5, and is similar to the type to be used in the smaller chambers.

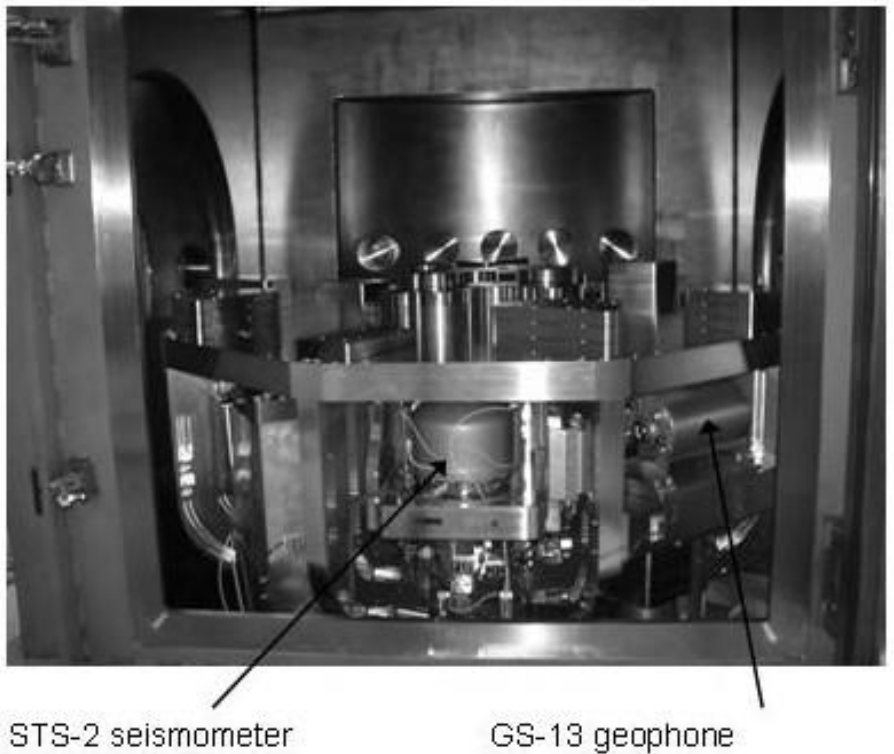

Figure 5. Picture of the Advanced LIGO seismic isolation technology demonstrator two-stage active platform inside the vacuum tank. The two stages are structurally interleaved to correctly position the centers of gravity. One of the 3 STS- 2 seismometers mounted on the first stage, and one of the Geotech GS-13 geophones mounted on stage two are indicated. The overall width is approximately $1.5 \mathrm{~m}$. Masses simulating the load of the suspension system can be seen on the optics table which is part of stage two of the platform.

Investigation of the performance of the technology demonstrator prototype is well underway. The overall control strategy is hierarchical in form, with active damping of the two stages being followed by closing feedback loops to control tilt. 
After tilt control is established, further control loops are closed to complete the control of stage one. Stage two control loops are subsequently brought into operation. The overall system will include 27 loops, and will use both sensor blending and sensor correction techniques, discussed in the previous section. Preliminary measurements from the first stage are shown in Fig. 6. In this figure, the noise levels on the ground and on the first stage are shown for one direction of horizontal motion. It can be seen that isolation is obtained above $1 \mathrm{~Hz}$, and at around $10 \mathrm{~Hz}$ there is an isolation factor of approximately 30. Results in the other horizontal direction and in the vertical direction are similar. A further factor of $\sim 30$ at $10 \mathrm{~Hz}$ is expected from stage two, which in conjunction with that of stage one should meet the overall isolation requirements for this system for Advanced LIGO. For the data shown below, the blend frequency between the relative position sensor and geophone was set at $0.5 \mathrm{~Hz}$ and sensor correction was not yet implemented, thus limiting the low frequency isolation performance. Techniques for improving low frequency isolation is the topic of another paper in this volume. $^{9}$

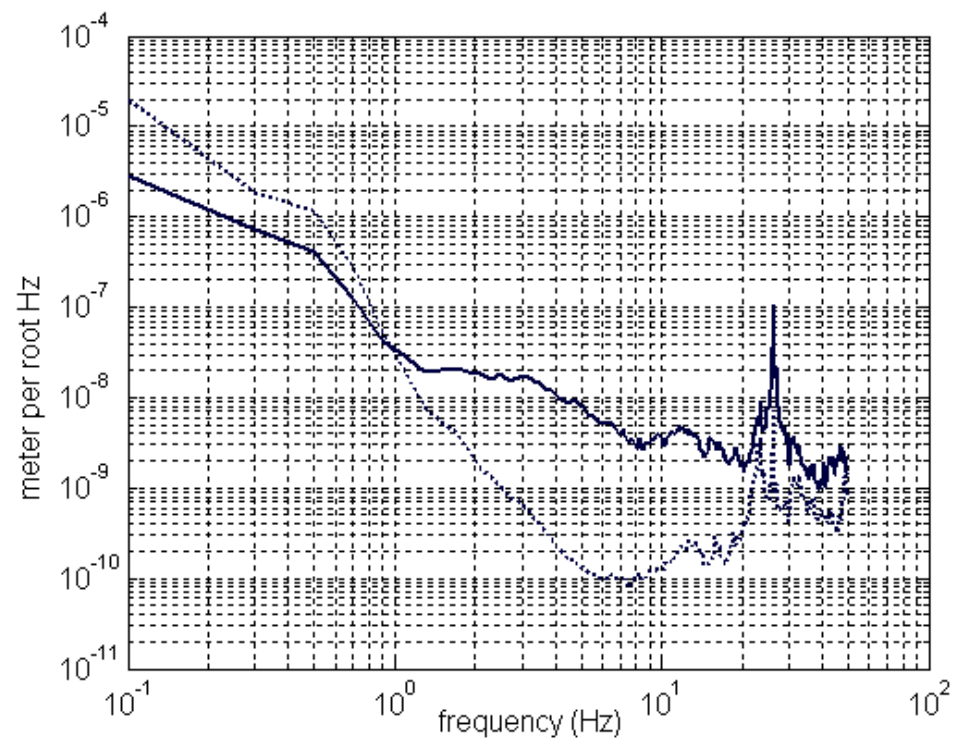

Figure 6. Measurements from the technology demonstrator prototype active isolation platform at Stanford. The heavy black curve shows the displacement noise level on the ground in a horizontal direction and the dotted curve the residual noise on stage 1 of the platform. The blend frequency used for these measurements was $0.5 \mathrm{~Hz}$. The feature seen at around $25 \mathrm{~Hz}$ is believed to be a resonance of the current support structure.

In parallel with the research effort underway to investigate the performance and optimize the control design of the technology demonstrator at Stanford, a new prototype is currently being designed for installation at LASTI, which will essentially be the design for Advanced LIGO. It is scheduled to be delivered in late 2004 .

\section{QUADRUPLE PENDULUM SUSPENSION DEVELOPMENT}

The suspension system design for Advanced LIGO is based on an extension of the triple pendulum design ${ }^{10,11}$ developed $^{10}$ for GEO 600 - the German/UK gravitational wave detector. The new design differs quite radically from the existing LIGO suspension design. The existing design has test masses (mirrors) hung as single pendulums on wire slings, with actuation for both damping (local control) of the pendulum modes and global control of the interferometer to hold it at its correct operating position being applied directly to the test masses via coil and magnet systems. The magnets are attached to the masses. The GEO 600 design and its extension for Advanced LIGO represent second-generation designs for which the performance is more aggressive than that currently used in LIGO, in particular in terms of the reduction of thermal noise associated with the suspension of the mirrors. The Advanced LIGO design has been chosen to aim to reach a target sensitivity corresponding to a residual noise displacement of $10^{-19} \mathrm{~m} / \sqrt{ } \mathrm{Hz}$ at $10 \mathrm{~Hz}$ at each of the test masses for each of the two main noise terms, suspension thermal noise and residual seismic noise. Other noise sources such as those due to the local and global control systems are required to lie below this. 
The main features of the proposed baseline design are as follows:

- $\quad$ Sapphire mirrors (40 kg) will form the lowest stage of a quadruple pendulum, and will be suspended on 4 vertical fused silica ribbons to reduce suspension thermal noise.

- The ribbons will be welded to fused silica "ears" or prisms which are silicate bonded ${ }^{12}$ to the flat sides of the penultimate mass and the mirror below. This technique ensures that the low mechanical loss of the mirror itself is preserved, maintaining the low thermal noise of the sapphire substrate.

- Included in the quadruple pendulum are three stages of cantilever blade springs made of maraging steel, similar to those used in the French-Italian Virgo gravitational wave detector ${ }^{13}$, to increase the vertical seismic isolation.

- $\quad$ The damping (local control) of all of the low frequency modes of the quadruple pendulum will be carried out by using 6 co-located sensors and actuators at the highest mass of the multiple pendulum, and may be supplemented by using eddy current damping applied at this mass for certain degrees of freedom. Thus noise associated with the local control is isolated by the stages below.

- Global longitudinal forces will be applied in a hierarchical feedback system via a quiet reaction pendulum, essentially identical in mechanical design to the main pendulum, but with wires replacing the silica ribbons. Large low frequency forces will be applied electromagnetically between the penultimate masses, and small higher frequency signals applied electrostatically between the mirror and the corresponding lowest reaction mass. That mass will be made of dense glass with a patterned gold coating.

With respect to the global control of the interferometer it is intended that in the final configuration the low frequency forces will be kept close to null by using the HEPI system described in section 2 .

Further details on design aspects of the quadruple pendulum suspension can be found elsewhere ${ }^{14}$. A schematic diagram is shown in Fig. 7.

The overall seismic isolation is the product of the isolation of the three sub-systems. The target residual noise level on the isolation platform is $2 \times 10^{-13} \mathrm{~m} / \sqrt{ } \mathrm{Hz}$ at $10 \mathrm{~Hz}$ in both longitudinal (i.e. parallel to the laser beam) and vertical directions. The expected transmissibility of the quadruple pendulum in the longitudinal direction (suitably damped) is around $3 \times 10^{-7}$ at $10 \mathrm{~Hz}$ (and falls off approximately as $1 / \mathrm{f}^{7}$ ). In the vertical direction the transmissibility is approximately 3 orders of magnitude higher, and this combined with an assumed vertical to longitudinal coupling factor of $10^{-3}$ gives a similar overall isolation. Combining the longitudinal and vertical contributions in quadrature, we see the overall target of $10^{-19} \mathrm{~m} / \sqrt{ } \mathrm{Hz}$ at $10 \mathrm{~Hz}$ for residual seismic noise should be met.

We now discuss some of the ongoing research and development on suspensions.

\subsection{Thermal noise issues}

The most crucial part of the suspension in terms of minimizing the suspension thermal noise is the final stage. Thermal noise from upper stages is filtered by the stages below, and providing the design of the upper stages is carefully chosen, the suspension thermal noise is expected to be dominated by the lowest stage at $10 \mathrm{~Hz}$ and above. The use of silica fibers in the final stage rather than steel wires gives an immediate improvement due to silica's lower intrinsic loss. A further reduction in noise is expected by using a ribbon cross-section oriented such that the bending loss is minimized in the direction along the axis of the interferometer arm. An estimate of the resulting thermal noise is shown in Fig. 8.

Experience of preparing and welding fibers and bonding ears has been gained in GEO. However these techniques require development in several areas for application in Advanced LIGO. Firstly there is a need to develop ear design to scale up from $10 \mathrm{~kg}$ to $40 \mathrm{~kg}$ mirrors. One wishes to achieve a design with the required mechanical strength and which minimizes the thermal noise associated with the bond at a level such that it makes an insignificant increase in the predicted overall noise level of the detector. An example of the stress in a finite element model of an ear under load is shown in Fig. 9. Finite element analysis is also currently being carried out on a model of a test mass with ears attached to study the strain energy distribution near the interfaces and hence estimate the thermal noise due to the bond.

Secondly a $100 \mathrm{~W} \mathrm{CO}_{2}$ laser system for pulling and welding of ribbons is being developed at the University of Glasgow. Advantages of such a system are high precision heat delivery, improved shape control, reduced contaminants and 
spatially selective annealing. The latter should reduce stresses and increase strength and reliability. In parallel a system for image capture and edge detection is also being developed to aid in ribbon profiling.
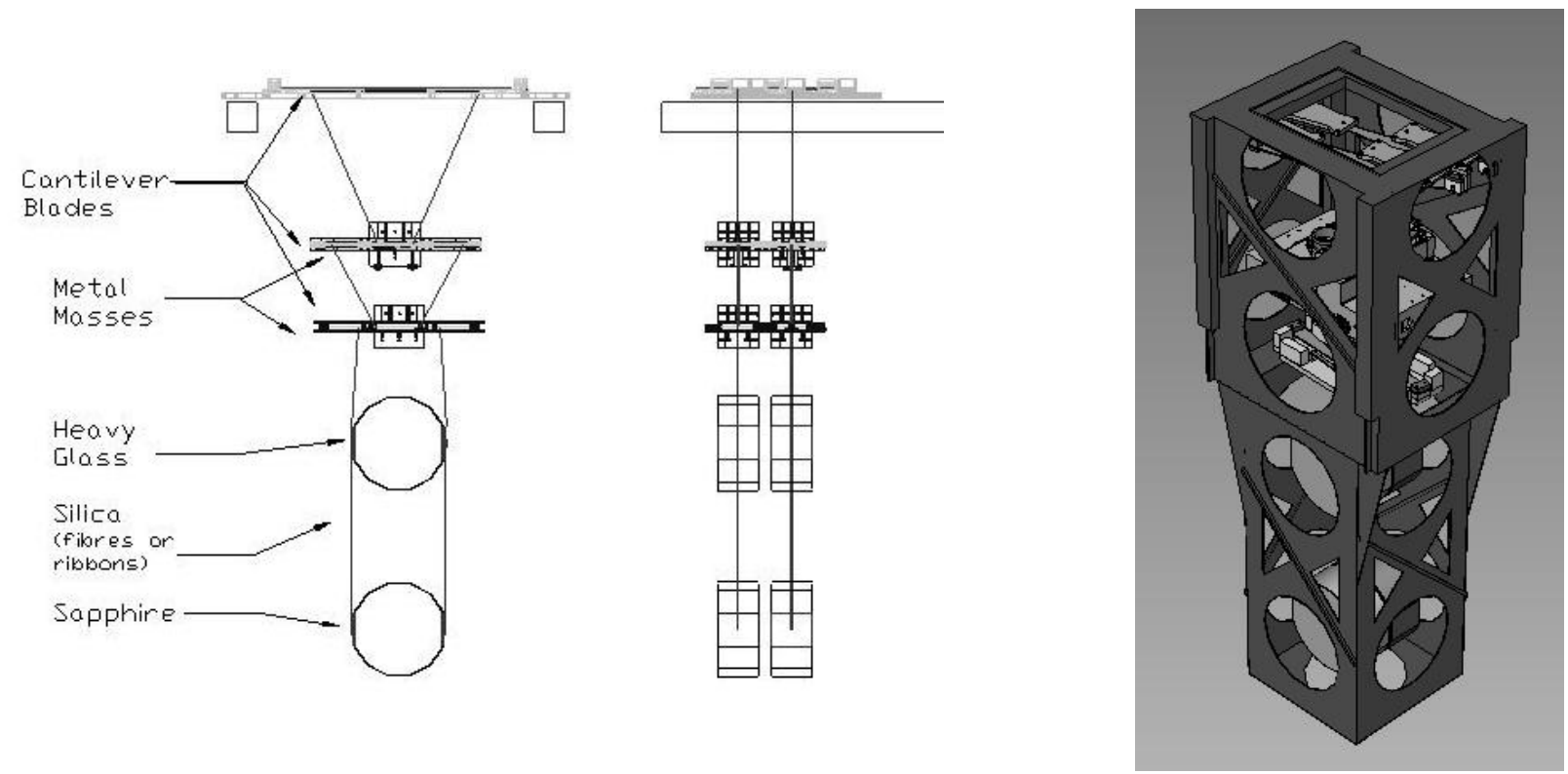

Figure 7. Schematic diagram of quadruple pendulum suspension system for Advanced LIGO. A face view of the main chain is shown on the left, and in the middle is shown a side view with main and reaction chains visible. On the right is a conceptual design for the supporting structure which is approximately $2 \mathrm{~m}$ in height, and whose top surface would be rigidly attached to the optics table. This structure would support elements of the sensor and actuators for local control, and earthquake stops to restrict motion of the masses and support them in the event of a wire or ribbon failure.

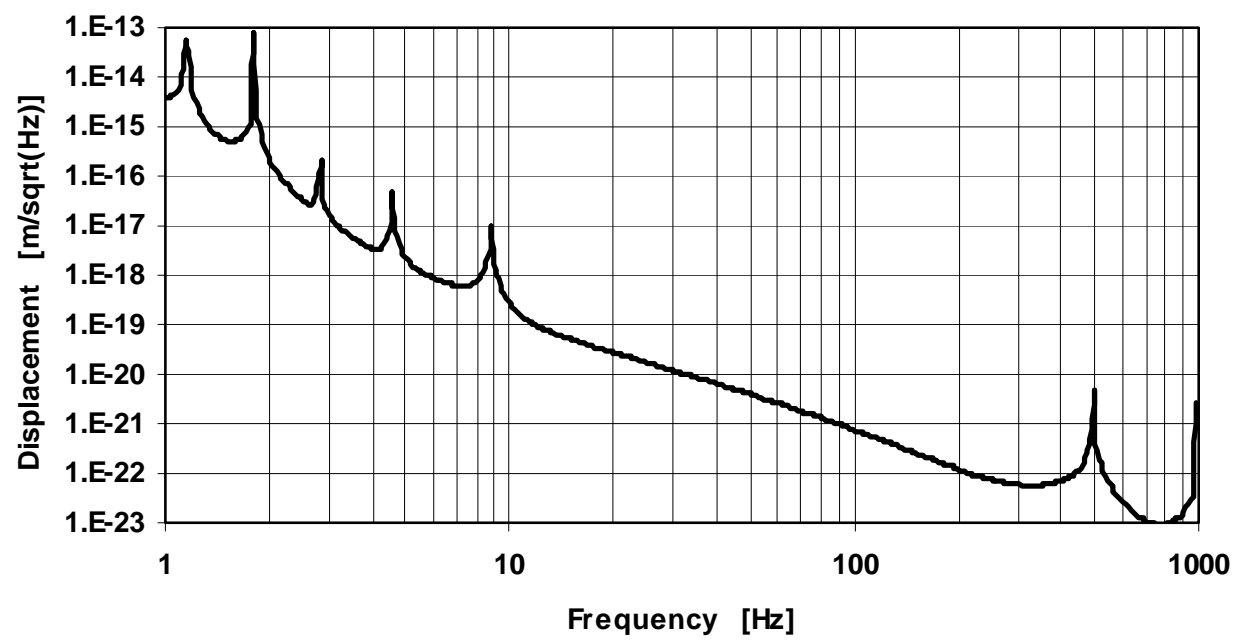

Figure 8. Estimate of suspension thermal noise for quadruple pendulum suspension with masses $22 \mathrm{~kg}, 22 \mathrm{~kg}, 40 \mathrm{~kg}, 40 \mathrm{~kg}$ (top to bottom). Optic is suspended on four $60 \mathrm{~cm}$ silica ribbons of dimensions $1.1 \mathrm{~mm}$ x $0.11 \mathrm{~mm}$.

Thirdly, investigations of the quality factors (Q) achievable for the pendulum and "violin" modes of a ribbon suspension are underway at Glasgow. To date a pendulum Q of $1.4 \times 10^{8}$ has been measured for a $43.5 \mathrm{~g}$ mass supported on a single ribbon of $405 \times 1.2 \times 0.11 \mathrm{~mm}$, compared to a theoretical value of $2.2 \times 10^{8}$. A Q of $1.5 \times 10^{8}$ has been measured for the 
lowest violin mode of a single ribbon of dimensions 420 x 1.12 x $0.12 \mathrm{~mm}$ supporting a $2.4 \mathrm{~kg}$ mass. The expected theoretical value was $7 \times 10^{8}$, and investigations are ongoing to establish what is limiting the experimental result.

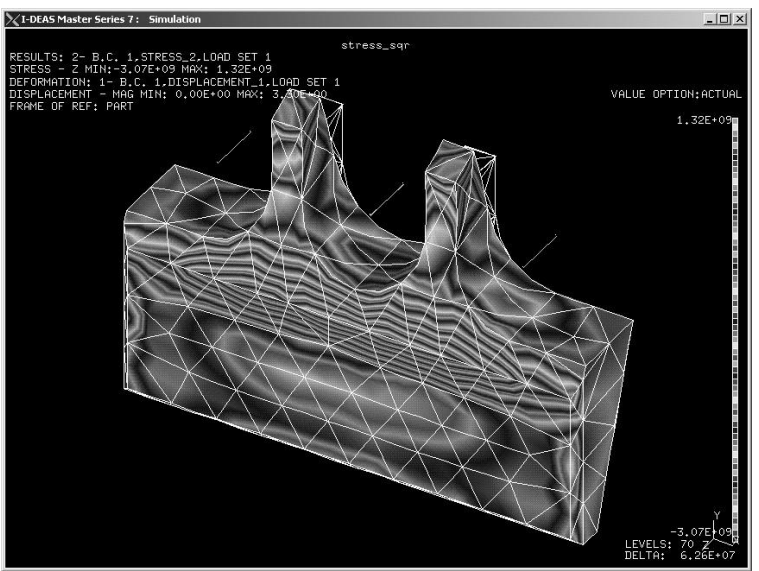

Figure 9. Finite element model of a GEO ear design under load. Closely spaced contours show rapidly varying stress which in this case typically occurs at regions of high stress.

\subsection{Control issues}

In the past few months there has been a major program of work in the UK to develop quiet sensors for use in the local control systems for the Advanced LIGO suspensions. Even allowing for the isolation achieved by carrying out sensing and actuation for local control at the top mass in the multiple suspensions (quadruple for the most sensitive optics) the noise performance is demanding. For example the current LIGO and GEO detectors use compact optical shadow sensors with a noise performance of around $10^{-10} \mathrm{~m} / \sqrt{ } \mathrm{Hz}$ at $10 \mathrm{~Hz}$. The requirement on residual noise due to the sensors at the most sensitive optics is to be $10^{-20} \mathrm{~m} / \sqrt{ } \mathrm{Hz}$. The isolation factor for residual sensor noise at $10 \mathrm{~Hz}$ is typically of order $10^{7}$. Thus assuming there is still substantial gain in the feedback loop at $10 \mathrm{~Hz}$, these numbers suggest that sensor noise needs to be approximately three orders of magnitude better than those in current use to meet the requirements. A promising low noise interferometric sensor illuminated with a diode laser has been developed. It uses polarizing optics to obtain quadrature outputs to allow fringe counting in addition to fractional fringe measurements. A bench-top version has already shown noise performance of $\sim 5 \times 10^{-13} \mathrm{~m} / \sqrt{\mathrm{Hz}}$ around $10 \mathrm{~Hz}$ with a dynamic range of $3 \mathrm{~mm}$, and further work on producing a compact mechanical design is currently underway. An alternative strategy involving combining active control using shadow sensors for the lowest frequency modes of the suspension with eddy current damping for the higher frequency modes, and possibly relaxing the amount of damping required once global control of the interferometer has been achieved, is also under consideration. Eddy current damping applied to the top mass of a triple pendulum suspension has already been demonstrated ${ }^{15}$.

\subsection{Mechanical design issues}

In Advanced LIGO there will be several types of suspensions, triple and quadruple, tailored to support the various types of optics which have different masses and sizes and different noise requirements. Modeling for all the main designs is already underway and all-metal triple pendulum prototypes for the modecleaner mirrors have already been constructed. Initial tests of the mode frequencies and local control have demonstrated the expected behavior. Currently we are developing the design for the quadruple pendulum suspension to be used for the $40 \mathrm{~kg}$ test masses, which is the most demanding both from the desired noise performance and the practical challenges presented in assembling the overall suspension plus structure. An ergonomic arm being used to handle a metal representation of a $40 \mathrm{~kg}$ sapphire test mass is shown in Fig. 10. In parallel with the development of the suspension itself, finite element analysis is being carried out on a conceptual design of support structure (shown in Fig. 7) in conjunction with an isolation platform to investigate modal frequencies and the behavior of the combined system.

Design of an all-metal prototype quadruple pendulum is well underway and it is expected to be constructed and have undergone preliminary investigation of mode frequencies and damping at Caltech by the end of 2004. After cleaning, the system with its support structure will be sent to MIT where it will be suspended from the LASTI active platform referred 
to in section 3 above. Experience on installation and alignment will be gained and investigation of the behavior of the two systems in combination will be carried out, with the results being compared to theoretical models

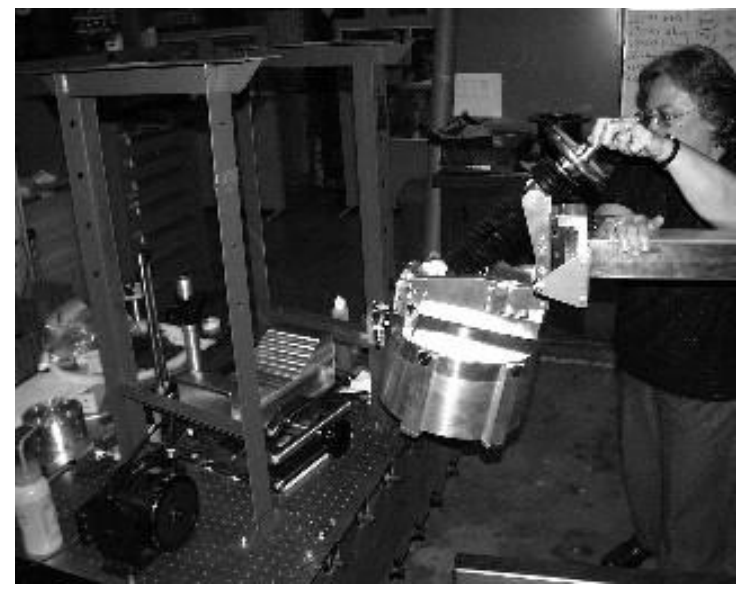

Figure 10. Ergonomic arm being used to manipulate a $40 \mathrm{~kg}$ mass in a controlled fashion.

\section{CONCLUSIONS}

We have presented an overview of the three sub-systems which are being developed to meet the alignment, isolation and suspension thermal noise requirements for Advanced LIGO. The HEPI system is currently being installed at the LIGO Livingston site and is expected to be in operation later this year. A HEPI system is also being fitted into a LIGO-sized chamber at LASTI. A technology demonstrator active isolation platform is currently under development and testing at Stanford. A new prototype for LASTI which is expected to be essentially the design for Advanced LIGO is currently under construction and should be delivered towards the end of 2004. It will be installed in the chamber already fitted out with the HEPI system. After stand-alone testing of the active isolation platform is carried out, the all metal quadruple pendulum prototype will be installed, and testing of the overall alignment, isolation and suspension system can then take place.

\section{ACKNOWLEDGEMENTS}

The authors gratefully acknowledge financial support from the following agencies and universities: National Science Foundation, Particle Physics and Astronomy Research Council, the Max-Planck Society, the Universities of Glasgow, Birmingham and Strathclyde. The work at Stanford University is supported by NSF grant PHY-0140297. The work at the LIGO Laboratories and Observatories is supported by NSF grant PHY-0107417. The work at Louisiana State University is supported by NSF grants PHY-0071316 and PHY-0304924 and Louisiana Board of Regents contract LEQSF(2000-03)-RD-A-06.

\section{REFERENCES}

1. B Barish and R Weiss, "LIGO and the Detection of Gravitational Waves", Phys. Today, 52, 44-50, 1999

2. The LIGO Scientific collaboration, B Abbott et al, "Detector Description and Performance for the First Coincidence Observations between LIGO and GEO" Nucl.Instrum.Meth. A517, 154-179, 2004

3. P Fritschel, "Second generation instruments for the Laser Interferometer Gravitational Wave Observatory (LIGO)" Gravitational-Wave Detection, M. Cruise, P. Saulson Eds. 4856, 282-291, SPIE, Bellingham, WA. 2003 
4. E Gustafson, D Shoemaker, K Strain and R Weiss, LSC White Paper on Detector Research and Development, LIGO document number T990080-00-D, at http://www.ligo.caltech.edu/docs/T/T990080-00.pdf

5. C Hardham, B Abbott, R Abbott, G Allen, R Bork, C Campbell, K Carter, D Coyne, D DeBra, T Evans, J Faludi, A Ganguli, J Giaime, M Hammond, W Hua, J Kern, J LaCour, B Lantz, M Macinnis, K Mailand, K Mason, R Mittleman, J Nichol, J van Niekerk, N Robertson, D Ottaway, H Overmier, J Phinney, B Rankin, D Sellers, P Sarin, D Shoemaker, O Spjeld, G Traylor, S Wen, M Zucker, "Multi-DOF Isolation and Alignment with Quiet Hydraulic Actuators", To be published in Proceedings of ASPE Spring Topical Meeting on Control of Precision Systems, April 2004, $127-132$

6. W Hua, D DeBra, C T Hardham, B T Lantz and J A Giaime, "Polyphase FIR Complementary Filters for Control Systems" To be published in Proceedings of ASPE Spring Topical Meeting on Control of Precision Systems, April 2004, page 109-114.

7. R Abbott, R Adhikari, G Allen, S Cowley, E Daw, D DeBra, J Giaime, G Hammond, M Hammond, C Hardham, J How, W Hua, W Johnson, B Lantz, K Mason, R Mittleman, J Nichol, S Richman, J Rollins, D Shoemaker, G Stapfer and R Stebbins, "Seismic isolation for Advanced LIGO”, Class. Quantum Grav. 19, 1591-1597, 2002

8. R Abbott, R Adhikari, G Allen, D Baglino, C Campbell, D Coyne, E Daw, D DeBra, J Faludi, P Fritschel, A Ganguli, J Giaime, M Hammond, C Hardham, G Harry, W Hua, L Jones, J Kern, B Lantz, K Lilienkamp, K Mailand, K Mason, R Mittleman, S Nayfeh, D Ottaway, J Phinney, W Rankin, N Robertson, R Scheffler, D H Shoemaker, S Wen, M Zucker and L Zuo, "Seismic isolation enhancements for initial and advanced LIGO", Class. Quantum Grav, 21, S915-S921, 2004

9. W Hua et al, "Low frequency isolation for Advanced LIGO", this volume

10. M V Plissi, C I Torrie, M E Husman, N A Robertson, K A Strain, H Ward, H Lück, J Hough, "GEO 600 triple pendulum suspension system: Seismic isolation and control", Rev. Sci. Instrum., 71, 2539 - 2545, 2000

11. G Cagnoli, L Gammaitoni, J Hough, J Kovalik, S McIntosh, M Punturo, S Rowan "Very High Q measurements on a Fused Silica Monolithic Pendulum for Use in Enhanced Gravity Wave Detectors", Phys. Rev. Lett. 85, 2442 - 2445 , 2000

12. S Rowan, S M Twyford, J Hough, D-H. Gwo and R Route, "Mechanical losses associated with the technique of hydroxide-catalysis bonding of fused silica", Phys. Lett. A, 246, 471-478, 1998

13. S Braccini, C Casciano, F Cordero, F Corvace, M De Sanctis, R Franco, F Frasconi, E Majorana, G Paparo, R Passaquieti, P Rapagnani, F Ricci, D Righetti, A Solina and R Valentini, "The maraging-steel blades of the Virgo super attenuator", Meas. Sci. Technol, 11, 467-476, 2000

14. N A Robertson, G Cagnoli, D R M Crooks, E Elliffe, J E Faller, P Fritschel, S Gossler, A Grant, A Heptonstall, J Hough, H Lück, R Mittleman, M Perreur-Lloyd, M V Plissi, S Rowan, D H Shoemaker, P H Sneddon, K A Strain, C I Torrie, H Ward and P Willems, "Quadruple suspension design for Advanced LIGO”, Class. Quantum Grav. 19, 40434058, 2002

15. M V Plissi, C I Torrie, M Barton, N A Robertson, A Grant, C A Cantley, K A Strain, P A Willems, J H Romie, K D Skeldon, M M Perreur-Lloyd, R A Jones and J Hough, “ An investigation of eddy-current damping of multi-stage pendulum suspensions for use in gravitational wave detectors, submitted to Rev Sci Instrum 